Published in final edited form as:

Curr Infect Dis Rep. 2012 October ; 14(5): 551-557. doi:10.1007/s11908-012-0284-2.

\title{
Prosthetic Joint Infections
}

\author{
Saima Aslam ${ }^{1}$ and Rabih O. Darouiche ${ }^{2}$ \\ ${ }^{1}$ Division of Infectious Diseases, Department of Medicine, University of California, San Diego, CA, \\ USA
}

${ }^{2}$ Center for Prostheses Infection, Michael E. DeBakey Veterans Affairs Medical Center and Baylor College of Medicine, Houston, TX, USA

\begin{abstract}
Prosthetic joint infections represent a major therapeutic challenge for both healthcare providers and patients. This paper reviews the predisposing factors, pathogenesis, microbiology, diagnosis, treatment and prophylaxis of prosthetic joint infection. The most optimal management strategy should be identified based on a number of considerations including type and duration of infection, antimicrobial susceptibility of the infecting pathogen, condition of infected tissues and bone stock, patient wishes and functional status.
\end{abstract}

\section{Keywords}

Prosthetic Joint Infections; Antimicrobial Prophylaxis; Resection arthroplasty; Long-term antimicrobial suppression

\section{Introduction}

Prosthetic joint infection (PJI) is a devastating complication of orthopedic joint implantation. The incidence of PJI is $1-2.5 \%$ for primary hip or knee replacements and $2.1-$ $5.8 \%$ for revision surgeries.[1-3] The cost of treating such an infection is estimated to be > $\$ 50,000$ per episode.[4] Revision procedures that are performed because of infection are associated with a longer operative time, more blood loss, greater number of complications, and increased healthcare costs than revision surgeries done for aseptic loosening or primary arthroplasty.[5] The mortality associated with surgical intervention for such an infection is low, but the degree of morbidity is quite overwhelming. Successful treatment of PJI is usually difficult and often involves multiple surgical interventions plus prolonged antibiotic course(s).

\section{Risk Factors}

Risk factors for PJI that have been identified in cohort or case control analyses include recent bacteremia, surgical site infection, trauma to the prosthetic joint, prior PJI, a National Nosocomial Infection Surveillance (NNIS) score of one or two, concurrent malignancy, prior total hip arthroplasty (THA), presence of constrained and hinged knee prosthesis, revision surgery, procedure duration, steroid therapy, obesity, presence of rheumatoid

Corresponding Author: Saima Aslam, MD, MS, 200 W. Arbor Drive, NARF 307, MC 8415, San Diego, CA 92103, Tel: (619) 543-6327, Fax: (619) 543-3383, saslam@ucsd.edu.

Disclosure

No potential conflicts of interest relevant to this article were reported. 
arthritis, recurrent urinary tract infections, diabetes mellitus, and lower socioeconomic status.[4, 6-14]

Once an infection is diagnosed, a number of therapeutic approaches may be adopted. The selection of a particular approach depends on host factors (age, baseline mobility, comorbidities, etc.), virulence and antimicrobial susceptibility of the infecting organism, duration of infection, prosthesis factors (stability of implant, loss of bone stock and soft tissue because of previous surgeries or infection) and patient expectation. In general, whenever feasible, it is recommended to remove the infected hardware, treat with antibiotics, and then undergo re-implantation. However, as discussed below, there are some clinical scenarios that warrant other types of surgical intervention.

\section{Pathogenesis and Microbiology}

An implant may be seeded with pathogenic organisms during surgery, in the peri-operative period or by means of bacteremia. Generally, early infections (i.e. within 3 months of the initial surgery) are due to peri-operative inoculation, whereas late infections tend to occur due to hematogenous seeding of the prosthesis or through compromised local tissues. Regardless of the method of joint contamination, the end result is production of biofilm that surrounds the prosthesis surface. A biofilm is composed of organisms adherent to the underlying prosthesis and encased within a polysaccharide matrix. It is characterized by low cell turnover, protection from host immune responses and antimicrobial resistance.[15, 16] The presence of the biofilm makes it difficult to treat such infections with antibiotics alone and, thus, surgery is usually required in order to cure the infection.

Over half of the cases of PJI are caused by staphylococci, including both Staphylococcus epidermidis and $S$. aureus.[17] Recent trends suggest that about half of the $S$. aureus isolates are resistant to methicillin, which are associated with a higher rate of treatment failure than methicillin-sensitive $S$. aureus (MSSA).[18, 19] Other bacteria and fungi make up the remainder of cases, with occasional episodes of mycobacterial infection. Approximately $20 \%$ of PJI cases are polymicrobial, generally involving staphylococci, and about $7 \%$ are culture negative.[20] Not unexpectedly, prior receipt of antimicrobials is associated with increased rate of culture negativity.[21]

\section{Diagnostic Methods}

Traditionally, the diagnosis of a PJI is made by the presence of at least one of the following criteria: (a) acute inflammation noted on histopathological examination of peri-prosthetic tissue, (b) presence of a sinus tract communicating with the prosthesis, (c) gross purulence in the joint space, and (d) isolation of the same organism from two or more cultures of joint aspirates or intra-operative peri-prosthetic tissue specimens.[20] To achieve optimal sensitivity, antibiotics should be discontinued at least 2 weeks prior to microbiologic testing. Cultures of sinus tract exudates should not be performed as they have poor correlation with surgical cultures and generally represent colonization of the tract.[20] At least 5 or 6 periprosthetic surgical specimens should be obtained to increase the yield.[22]

A recent clinical trial showed that sonication of the prosthesis followed by culture of the sonicate fluid is more sensitive than culture of peri-prosthetic tissue $(78.5 \%$ vs. $60.8 \%, \mathrm{p}$ $<0.001)$.[17] This test, however, has not been widely implemented in clinical laboratories as of yet. Another method to increase the diagnostic yield of microbiologic specimens is to inoculate joint aspirate fluid into blood culture bottles rather than directly plating them on agar plates.[23] Subclinical infection, diagnosed by a positive culture of polymethylmethacrylate (PMMA) cement spacer sonicate, prior to undergoing the second 
stage of a two-stage exchange for a PJI was associated with clinical failure of the replacement prosthesis.[24]

C-reactive protein (CRP) and erythrocyte sedimentation rate (ESR) have been clinically utilized to help support the diagnosis of a PJI. A recent study analyzed pre-operative CRP and ESR prior to removal of the orthopedic implant and found that CRP was significantly different in patients with aseptic failure vs. infection of the knee, hip, shoulder and spinal implants.[25] ESR was significantly elevated in patients with infections of the knee, hip and spinal implants but not in those with shoulder arthroplasties. The combination of normal CRP and ESR predicted the absence of infection in 94, 94 and $91 \%$ of patients embarking on removal of the knee, hip or spinal implant, respectively.[25] However, the levels of CRP and ESR prior to the second surgery of a two-stage exchange because of a previously infected knee arthroplasty did not exhibit a predictive pattern indicative of infection. Synovial fluid WBC count was not predictive of persistent infection either. [26]

There are conflicting reports regarding improved diagnostic accuracy of PJI with the use of various molecular techniques. According to a recent publication, 16S rRNA gene real-time PCR of peri-prosthetic samples did not identify more cases of septic prosthetic loosening than did culture of adequate periprosthetic biopsies.[27] PCR-hybridization of sonicate fluid increased the microbiologic yield by $10 \%$ in another study, but it is unclear if that findings reflects a true enhancement in the diagnosis of infection or represent false positive results. [28]

\section{Management Strategies}

The primary goal of treatment is a pain-free and functional joint. Prosthesis stability is a prerequisite for achieving this goal and, in general, an unstable prosthesis will need to be replaced. Once an infection is diagnosed, various therapeutic strategies could be considered. The choice of a particular approach depends on host factors (age, baseline mobility, comorbidities), virulence and antimicrobial susceptibility of the infecting organism, duration of symptoms, and prosthesis factors (stability of implant, condition of peri-prosthetic bone and tissue). In general, it is recommended to remove the infected hardware whenever indicated and feasible, treat with antibiotics, and then undergo re-implantation. Figure 1 shows a suggested algorithm for the management of an infected prosthetic joint.[20]

Various strategies of surgical management have been utilized over the years. Below are the most commonly utilized strategies listed in the order of decreasing invasiveness:

\section{(1) Two-stage exchange}

This most commonly utilized approach has been the traditional gold standard in the treatment of infected total hip and knee arthroplasties for several decades. [29, 30] When embarking on a two-stage revision approach, the infected prosthetic joint (and cement, if present) is first surgically removed and antibiotic-impregnated joint spacer that contains PMMA is usually placed. Bioresorbable polymers, such as silica-calcium phosphate nanocomposite and micro-porous calcium phosphate, are being investigated as a novel method of local antibiotic delivery.[31, 32] In patients with extensive infection, antibioticimpregnated beads may be used as well. After treatment for 4-6 weeks with systemic antibiotics that are chosen based on antimicrobial susceptibilities, the joint spacer is removed and a new prosthetic joint is placed. [29, 30, 33, 34] This two-stage protocol is successful in treating 84-96\% of patients with infected THA [35-39] and 84-97\% of subjects with infected total knee arthroplasty (TKA).[39-41] 
Unfortunately, treatment regimens for infection by resistant organisms are less successful. Of 38 patients with methicillin-resistant $S$. aureus (MRSA) infection of THA who were treated with a two-stage exchange, $21 \%$ had recurrent infection requiring further revision surgery.[19] Use of antibiotic loaded articulating spacers in the case of infected TKA is associated with $88 \%$ cure rate and improvement in quality of life measures.[42]

A few studies have examined the role of a shorter duration of intravenous antibiotics between the two stages in an effort to shorten hospital stay, decrease medical costs, and enhance patient discomfort and mobility. Placement of an antibiotic loaded cement spacer during the first surgery followed by a 1-2 week course of intravenous antibiotics between the two surgeries allowed successful treatment (89-93\%) of infected THA.[43, 44]

The role of joint aspiration and/or intra-operative frozen section prior to reimplantation has not been systematically studied. Retrospective analyses of the role of intra-operative frozen sections at the time of reimplantation in patients with previously infected joint arthroplasties displayed a sensitivity of $25 \%$ and a specificity of $98 \%$ in diagnosing persistent infection. [45] Another approach, consisting of joint aspiration four weeks after the completion of a six-week course of intravenous antibiotics following resection arthroplasty for infected prosthetic knee joints, led to positive pre-revision cultures in 3/34 patients.[46] These patients were retreated with another six-week course of intravenous antibiotics prior to revision surgery and none developed a subsequent infection. False negative results are common if joint aspiration is done within two weeks of completing the antibiotic course.[47]

\section{(2) One-stage exchange}

This strategy comprises removal of the prosthetic joint and cement, thorough debridement of infected tissues, and the placement of a new prosthesis during the same surgery. A recent publication prospectively studied 84 patients with infected THA who underwent either a one-stage exchange $(n=38)$ or two-stage exchange $(n=46)$. A one-stage exchange was performed if the infecting organism was known prior to the surgery and bone loss on preoperative imaging was considered minor by the surgeon. All patients received six weeks of intravenous antibiotics followed by six weeks of oral antibiotics. The initial infection was cured in 38 patients $(100 \%)$ in the one-stage group and all but one patient $(97.8 \%)$ in the two-stage group.[48]

\section{(3) Debridement and retention of the infected implant}

This plan is generally associated with a poor success rate. Recent retrospective reviews of 246 cases of PJI noted an overall failure rate of 57-81\%. [49-51] The presence of staphylococci (which were the predominant type of infections) and type of treatment (retention vs. removal) predicted failure.[49, 51] However, details of antibiotic regimens were not routinely available. Generally, debridement and retention of the prosthesis is advisable only in a carefully selected patient population (as delineated in Figure 1). The likelihood of a successful debridement and retention approach is higher in patients with a short duration of symptoms prior to surgery (ranging between 2-21 days), a stable and functioning prosthesis, absence of a sinus tract, duration of infection within 30 days of the index arthroplasty, and infection by organisms other than $S$. aureus, especially MRSA. [5260] A three-month (for hip arthroplasty) to six-month (for knee arthroplasty) oral course of combination of a quinolone (or another oral antibiotic that is active against MRSA) and rifampin (retains its antimicrobial activity against biofilm-embedded organisms more so than most other antibiotics), if possible, is advisable in such cases. The efficacy of this approach was shown in a randomized clinical trial had a success rate with of $100 \%$ among recipients of ciprofloxacin/rifampin vs. 58\% in the ciprofloxacin/placebo group.[61] Even 
though the study used ciprofloxacin, we believe that any quinolone that is active against a particular $S$. aureus isolate can be used.

\section{(4) Long-term antimicrobial suppression}

This approach, which is not curative in intent, is recommended only in patients who are not surgical candidates. Use of rifampin, if possible, is recommended in combination with another active agent such as a quinolone, trimethoprim-sulfamethoxazole, and minocycline, to prevent development of rifampin resistance when used alone.[62-64]

\section{(5) Fusion}

When infection control is not feasible, at times the entire prosthesis is removed, and the joint is fused, such as in a knee arthrodesis.[20] This procedure is preferable than resection arthroplasty as patients can still remain ambulatory but, does require preservation of bonestock.

\section{(6) Resection arthroplasty}

In extreme cases when infection is not controllable by the means described above, a resection arthroplasty may be performed. This procedure comprises excision of all foreign and infected material and the joint space is allowed to fill with scar tissue. Most patients generally remain non-ambulatory.

The Infectious Disease Society of America (IDSA) clinical practice guidelines for the diagnosis and management of prosthetic joint infections are being finalized.

\section{Antimicrobial Prophylaxis}

Advisory statements from the American Association of Orthopaedic Surgeons and National Surgical Infection Prevention Project recommend routine antimicrobial prophylaxis for joint replacement surgery to minimize the risk of infection. $[65,66]$ Cefazolin or cefuroxime are routinely used with the recommendation that vancomycin could be administered in patients with $\beta$-lactam allergy, those with colonization by MRSA, or at high risk of developing an MRSA infection. Prophylactic antibiotic should be started within 1 hour (unless the infusion period of the selected antibiotic is much longer) of the surgical incision and completed within 24 hours. A recent paper highlighted the fact that in $63 \%$ of 163 patients with prosthetic joint infections developing mainly within 3 months of the index arthroplasty, the infecting organism was resistant to the antibiotic used for surgical prophylaxis.[18] In this study, MRSA was isolated from $45 \%$ of all cases of PJI. The area of antimicrobial prophylaxis for joint implantation is evolving and local epidemiologic patterns of infection are useful when making institutional recommendations.

The use of antimicrobial prophylaxis prior to invasive procedures in patients with indwelling arthroplasty is controversial. The American Academy of Orthopedic Surgeons published an information statement in 2009 which recommends that clinicians consider antibiotic prophylaxis for all total joint replacement patients prior to any invasive procedure that may cause bacteremia.[67] A position paper from the American Academy of Oral Medicine noted that the risk of patients' experiencing drug reactions or drug-resistant bacterial infections and the cost of antibiotic medications alone do not justify the recommendation for dental patients.[68] A recent case control study concluded that dental procedures were not risk factors for subsequent total hip or knee PJI and that the use of antibiotic prophylaxis prior to dental procedures did not decrease the risk of subsequent total hip or knee infection. [69] 


\section{Conclusions}

The infected prosthetic joint remains a challenging problem. The morbidity associated with this infection is high, management is expensive, and it can lead to a long and difficult course of recovery. The management of each patient needs to be individualized. Recent advances in the diagnosis and treatment, as highlighted in this review, have the potential of improving outcome of PJI.

\section{References}

Papers of particular interest, published recently, have been highlighted as **

1. Lentino JR. Prosthetic joint infections: bane of orthopedists, challenge for infectious disease specialists. Clin Infect Dis. 2003; 36(9):1157-61. [PubMed: 12715311]

2. Blom AW, Brown J, Taylor AH, et al. Infection after total knee arthroplasty. J Bone Joint Surg Br. 2004; 86(5):688-91. [PubMed: 15274264]

3. Blom AW, Taylor AH, Pattison G, et al. Infection after total hip arthroplasty. The Avon experience. J Bone Joint Surg Br. 2003; 85(7):956-9. [PubMed: 14516026]

4. Berbari EF, Hanssen AD, Duffy MC, et al. Risk factors for prosthetic joint infection: case-control study. Clin Infect Dis. 1998; 27(5):1247-54. [PubMed: 9827278]

5. Bozic KJ, Ries MD. The impact of infection after total hip arthroplasty on hospital and surgeon resource utilization. J Bone Joint Surg Am. 2005; 87(8):1746-51. [PubMed: 16085614]

6. Poss R, Thornhill TS, Ewald FC, et al. Factors influencing the incidence and outcome of infection following total joint arthroplasty. Clin Orthop Relat Res. 1984; (182):117-26. [PubMed: 6692605]

7. Kaandorp CJ, Dinant HJ, van de Laar MA, et al. Incidence and sources of native and prosthetic joint infection: a community based prospective survey. Ann Rheum Dis. 1997; 56(8):470-5. [PubMed: 9306869]

8. Wilson MG, Kelley K, Thornhill TS. Infection as a complication of total knee-replacement arthroplasty. Risk factors and treatment in sixty-seven cases. J Bone Joint Surg Am. 1990; 72(6): 878-83. [PubMed: 2365721]

9. Bongartz T, Halligan CS, Osmon DR, et al. Incidence and risk factors of prosthetic joint infection after total hip or knee replacement in patients with rheumatoid arthritis. Arthritis Rheum. 2008; 59(12):1713-20. [PubMed: 19035425]

10. Choong PF, Dowsey MM, Carr D, et al. Risk factors associated with acute hip prosthetic joint infections and outcome of treatment with a rifampinbased regimen. Acta Orthop. 2007; 78(6):75565. [PubMed: 18236181]

11. Aslam S, Reitman C, Darouiche RO. Risk factors for subsequent diagnosis of prosthetic joint infection. Infect Control Hosp Epidemiol. 31(3):298-301. [PubMed: 20088697]

12. Jamsen E, Huhtala H, Puolakka T, Moilanen T. Risk factors for infection after knee arthroplasty. A register-based analysis of 43,149 cases. J Bone Joint Surg Am. 2009; 91(1):38-47. [PubMed: 19122077]

13. Ong KL, Kurtz SM, Lau E, et al. Prosthetic joint infection risk after total hip arthroplasty in the Medicare population. J Arthroplasty. 2009; 24(6 Suppl):105-9. [PubMed: 19493644]

14. Font-Vizcarra L, Tornero E, Bori G, et al. Relationship between intraoperative cultures during hip arthroplasty, obesity, and the risk of early prosthetic joint infection: a prospective study of 428 patients. The International journal of artificial organs. 2011;34(9):870-5. Epub 2011/11/19. [PubMed: 22094568]

15. Aslam S. Effect of antibacterials on biofilms. American journal of infection control. 2008; 36(10):S175 e9-11. Epub 2008/12/17. [PubMed: 19084156]

**16. Zimmerli W, Moser C. Pathogenesis and treatment concepts of orthopaedic biofilm infections. FEMS immunology and medical microbiology. 2012; 65(2):158-68. Epub 2012/02/09.

[PubMed: 22309166] 
17. Trampuz A, Piper KE, Jacobson MJ, et al. Sonication of removed hip and knee prostheses for diagnosis of infection. The New England journal of medicine. 2007; 357(7):654-63. Epub 2007/08/19. [PubMed: 17699815]

18. Peel TN, Cheng AC, Buising KL, Choong PF. Microbiological aetiology, epidemiology, and clinical profile of prosthetic joint infections: are current antibiotic prophylaxis guidelines effective? Antimicrobial agents and chemotherapy. 2012; 56(5):2386-91. Epub 2012/02/09. [PubMed: 22314530]

19. Leung F, Richards CJ, Garbuz DS, et al. Two-stage total hip arthroplasty: how often does it control methicillin-resistant infection? Clinical orthopaedics and related research. 2011; 469(4):1009-15. Epub 2010/12/17. [PubMed: 21161741]

**20. Del Pozo JL, Patel R. Clinical practice. Infection associated with prosthetic joints. The New England journal of medicine. 2009; 361(8):787-94. Epub 2009/08/21. [PubMed: 19692690]

21. Malekzadeh D, Osmon DR, Lahr BD, et al. Prior use of antimicrobial therapy is a risk factor for culture-negative prosthetic joint infection. Clinical orthopaedics and related research. 2010; 468(8):2039-45. Epub 2010/04/20. [PubMed: 20401555]

22. Atkins BL, Athanasou N, Deeks JJ, et al. Prospective evaluation of criteria for microbiological diagnosis of prosthetic-joint infection at revision arthroplasty. The OSIRIS Collaborative Study Group. Journal of clinical microbiology. 1998; 36(10):2932-9. Epub 1998/09/17. [PubMed: 9738046]

23. Hughes JG, Vetter EA, Patel R, et al. Culture with BACTEC Peds Plus/F bottle compared with conventional methods for detection of bacteria in synovial fluid. Journal of clinical microbiology. 2001; 39(12):4468-71. Epub 2001/11/29. [PubMed: 11724863]

24. Sorli L, Puig L, Torres-Claramunt R, et al. The relationship between microbiology results in the second of a two-stage exchange procedure using cement spacers and the outcome after revision total joint replacement for infection: the use of sonication to aid bacteriological analysis. The Journal of bone and joint surgery British volume. 2012; 94(2):249-53. Epub 2012/02/11. [PubMed: 22323695]

25. Piper KE, Fernandez-Sampedro M, Steckelberg KE, et al. C-reactive protein, erythrocyte sedimentation rate and orthopedic implant infection. PloS one. 2010; 5(2):e9358. Epub 2010/02/25. [PubMed: 20179760]

26. Kusuma SK, Ward J, Jacofsky M, et al. What is the role of serological testing between stages of two-stage reconstruction of the infected prosthetic knee? Clinical orthopaedics and related research. 2011; 469(4):1002-8. Epub 2010/10/14. [PubMed: 20941647]

27. Bjerkan G, Witso E, Nor A, et al. A comprehensive microbiological evaluation of fifty-four patients undergoing revision surgery due to prosthetic joint loosening. Journal of medical microbiology. 2012; 61(Pt 4):572-81. Epub 2011/12/03. [PubMed: 22135023]

28. Esteban J, Alonso-Rodriguez N, Del-Prado G, et al. PCR-hybridization after sonication improves diagnosis of implant-related infection. Acta orthopaedica. 2012; 83(3):299-304. Epub 2012/05/24 [PubMed: 22616742]

29. Brause, BD. Infections with Prostheses in Bones and Joints. In: Gerald, M.; Mandell, JEB.; Dolin, Raphael, editors. Mandell, Douglas and Bennet's Principles and Practice of Infectious Diseases. 6. 2005. p. 1332-47.

30. Karchmer, AW. Infections in a Prosthetic Device. Gorbach, SLBJ.; Blacklow, NR., editors. Philadelphia: WB Saunders; 1998. p. 1666-78.

31. El-Ghannam A, Jahed K, Govindaswami M. Resorbable bioactive ceramic for treatment of bone infection. Journal of biomedical materials research Part A. 2010; 94(1):308-16. Epub 2010/02/27. [PubMed: 20186734]

32. Kazemzadeh-Narbat M, Kindrachuk J, Duan K, et al. Antimicrobial peptides on calcium phosphate-coated titanium for the prevention of implant-associated infections. Biomaterials. 2010; 31(36):9519-26. Epub 2010/10/26. [PubMed: 20970848]

33. Lieberman JR, Callaway GH, Salvati EA, et al. Treatment of the infected total hip arthroplasty with a two-stage reimplantation protocol. Clin Orthop Relat Res. 1994; (301):205-12. [PubMed: 8156676] 
34. McDonald DJ, Fitzgerald RH Jr, Ilstrup DM. Two-stage reconstruction of a total hip arthroplasty because of infection. J Bone Joint Surg Am. 1989; 71(6):828-34. [PubMed: 2745478]

35. Hanssen AD, Rand JA. Evaluation and treatment of infection at the site of a total hip or knee arthroplasty. Journal of Bone and Joint Surgery Am. 1998; 80-A(6):910-22.

36. Colyer RA, Capello WN. Surgical treatment of the infected hip implant. Two-stage reimplantation with a one-month interval. Clin Orthop Relat Res. 1994; (298):75-9. [PubMed: 8118998]

37. Sanchez-Sotelo J, Berry DJ, Hanssen AD, Cabanela ME. Midterm to long-term followup of staged reimplantation for infected hip arthroplasty. Clin Orthop Relat Res. 2009; 467(1):219-24. [PubMed: 18813895]

38. Masri BA, Panagiotopoulos KP, Greidanus NV, et al. Cementless two-stage exchange arthroplasty for infection after total hip arthroplasty. J Arthroplasty. 2007; 22(1):72-8. [PubMed: 17197311]

39. Bejon P, Berendt A, Atkins BL, et al. Two-stage revision for prosthetic joint infection: predictors of outcome and the role of reimplantation microbiology. J Antimicrob Chemother. 65(3):569-75. [PubMed: 20053693]

40. Hanssen AD, Trousdale RT, Osmon DR. Patient outcome with reinfection following reimplantation for the infected total knee arthroplasty. Clin Orthop Relat Res. 1995; (321):55-67. [PubMed: 7497686]

41. Haleem AA, Berry DJ, Hanssen AD. Mid-term to long-term followup of two-stage reimplantation for infected total knee arthroplasty. Clin Orthop Relat Res. 2004; (428):35-9. [PubMed: 15534516]

42. Gooding CR, Masri BA, Duncan CP, et al. Durable infection control and function with the PROSTALAC spacer in two-stage revision for infected knee arthroplasty. Clinical orthopaedics and related research. 2011; 469(4):985-93. Epub 2010/09/30. [PubMed: 20878287]

43. Hsieh PH, Huang KC, Lee PC, Lee MS. Two-stage revision of infected hip arthroplasty using an antibiotic-loaded spacer: retrospective comparison between short-term and prolonged antibiotic therapy. J Antimicrob Chemother. 2009; 64(2):392-7. [PubMed: 19477889]

44. Whittaker JP, Warren RE, Jones RS, Gregson PA. Is prolonged systemic antibiotic treatment essential in two-stage revision hip replacement for chronic Gram-positive infection? The Journal of bone and joint surgery British volume. 2009; 91(1):44-51. Epub 2008/12/19. [PubMed: 19092003]

45. Della Valle CJ, Bogner E, Desai P, et al. Analysis of frozen sections of intraoperative specimens obtained at the time of reoperation after hip or knee resection arthroplasty for the treatment of infection. J Bone Joint Surg Am. 1999; 81(5):684-9. [PubMed: 10360696]

46. Mont MA, Waldman BJ, Hungerford DS. Evaluation of preoperative cultures before second-stage reimplantation of a total knee prosthesis complicated by infection. A comparison-group study. $\mathrm{J}$ Bone Joint Surg Am. 2000; 82-A(11):1552-7. [PubMed: 11097443]

47. Lonner JH, Siliski JM, Della Valle C, et al. Role of knee aspiration after resection of the infected total knee arthroplasty. Am J Orthop. 2001; 30(4):305-9. [PubMed: 11334452]

48. Klouche S, Leonard P, Zeller V, et al. Infected total hip arthroplasty revision: one- or two-stage procedure? Orthopaedics \& traumatology, surgery \& research: OTSR. 2012; 98(2):144-50. Epub 2012/03/01

49. Koyonos L, Zmistowski B, Della Valle CJ, Parvizi J. Infection control rate of irrigation and debridement for periprosthetic joint infection. Clinical orthopaedics and related research. 2011; 469(11):3043-8. Epub 2011/05/10. [PubMed: 21553171]

50. Gardner J, Gioe TJ, Tatman P. Can this prosthesis be saved?: implant salvage attempts in infected primary TKA. Clinical orthopaedics and related research. 2011; 469(4):970-6. Epub 2010/06/15. [PubMed: 20544317]

51. Choi HR, von Knoch F, Zurakowski D, et al. Can implant retention be recommended for treatment of infected TKA? Clinical orthopaedics and related research. 2011; 469(4):961-9. Epub 2010/11/17. [PubMed: 21080127]

52. Brandt CM, Sistrunk WW, Duffy MC, et al. Staphylococcus aureus prosthetic joint infection treated with debridement and prosthesis retention. Clin Infect Dis. 1997; 24(5):914-9. [PubMed: 9142792] 
53. Meehan AM, Osmon DR, Duffy MC, et al. Outcome of penicillin-susceptible streptococcal prosthetic joint infection treated with debridement and retention of the prosthesis. Clin Infect Dis. 2003; 36(7):845-9. [PubMed: 12652384]

54. Crockarell JR, Hanssen AD, Osmon DR, Morrey BF. Treatment of infection with debridement and retention of the components following hip arthroplasty. J Bone Joint Surg Am. 1998; 80(9):130613. [PubMed: 9759815]

55. Schoifet SD, Morrey BF. Treatment of infection after total knee arthroplasty by debridement with retention of the components. J Bone Joint Surg Am. 1990; 72(9):1383-90. [PubMed: 2229118]

56. Tattevin P, Cremieux AC, Pottier P, et al. Prosthetic joint infection: when can prosthesis salvage be considered? Clin Infect Dis. 1999; 29(2):292-5. [PubMed: 10476729]

57. Teeny SM, Dorr L, Murata G, Conaty P. Treatment of infected total knee arthroplasty. Irrigation and debridement versus two-stage reimplantation. The Journal of arthroplasty. 1990; 5(1):35-9. Epub 1990/03/01. [PubMed: 2319246]

58. Mont MA, Waldman B, Banerjee C, et al. Multiple irrigation, debridement, and retention of components in infected total knee arthroplasty. The Journal of arthroplasty. 1997; 12(4):426-33. Epub 1997/06/01. [PubMed: 9195319]

59. Byren I, Bejon P, Atkins BL, et al. One hundred and twelve infected arthroplasties treated with 'DAIR' (debridement, antibiotics and implant retention): antibiotic duration and outcome. The Journal of antimicrobial chemotherapy. 2009; 63(6):1264-71. Epub 2009/04/02. [PubMed: 19336454]

60. Salgado CD, Dash S, Cantey JR, Marculescu CE. Higher risk of failure of methicillin-resistant Staphylococcus aureus prosthetic joint infections. Clinical orthopaedics and related research. 2007; 461:48-53. Epub 2007/05/31. [PubMed: 17534195]

61. Zimmerli W, Widmer AF, Blatter M, et al. Role of rifampin for treatment of orthopedic implantrelated staphylococcal infections: a randomized controlled trial. Foreign-Body Infection (FBI) Study Group. JAMA: the journal of the American Medical Association. 1998; 279(19):1537-41. Epub 1998/05/30. [PubMed: 9605897]

62. Pavoni GL, Giannella M, Falcone M, et al. Conservative medical therapy of prosthetic joint infections: retrospective analysis of an 8-year experience. Clinical microbiology and infection: the official publication of the European Society of Clinical Microbiology and Infectious Diseases. 2004; 10(9):831-7. Epub 2004/09/10. [PubMed: 15355415]

63. Rao N, Crossett LS, Sinha RK, Le Frock JL. Long-term suppression of infection in total joint arthroplasty. Clinical orthopaedics and related research. 2003; (414):55-60. Epub 2003/09/11. [PubMed: 12966277]

64. Widmer AF, Gaechter A, Ochsner PE, Zimmerli W. Antimicrobial treatment of orthopedic implant-related infections with rifampin combinations. Clinical infectious diseases: an official publication of the Infectious Diseases Society of America. 1992; 14(6):1251-3. Epub 1992/06/01. [PubMed: 1623081]

65. American Association of Orthopaedic Surgeons. Recommendations for the Use of Intravenous Antibiotic Prophylaxis in Primary Total Joint Arthroplasty. 2004. [June 27, 2012]; Available from: http://www6.aaos.org/news/PDFopen/PDFopen.cfm?page_url=http://www.aaos.org/about/papers/ advistmt/1027.asp

66. Bratzler DW, Houck PM. Antimicrobial prophylaxis for surgery: an advisory statement from the National Surgical Infection Prevention Project. Clinical infectious diseases: an official publication of the Infectious Diseases Society of America. 2004; 38(12):1706-15. Epub 2004/07/01. [PubMed: 15227616]

67. American Association of Orthopaedic Surgeons. Antibiotic Prophylaxis for Bacteremia in Patients with Joint Replacements. 2009. [cited 2012 June 24, 2012]; Available from: http://www.aaos.org/about/papers/advistmt/1033.asp

68. Little JW, Jacobson JJ, Lockhart PB. The dental treatment of patients with joint replacements: a position paper from the American Academy of Oral Medicine. J Am Dent Assoc. 2010; 141(6): 667-71. Epub 2010/06/03. [PubMed: 20516097]

**69. Berbari EF, Osmon DR, Carr A, et al. Dental procedures as risk factors for prosthetic hip or knee infection: a hospital-based prospective case-control study. Clinical infectious diseases: an 
official publication of the Infectious Diseases Society of America. 2010; 50(1):8-16. Epub 2009/12/03. [PubMed: 19951109] 


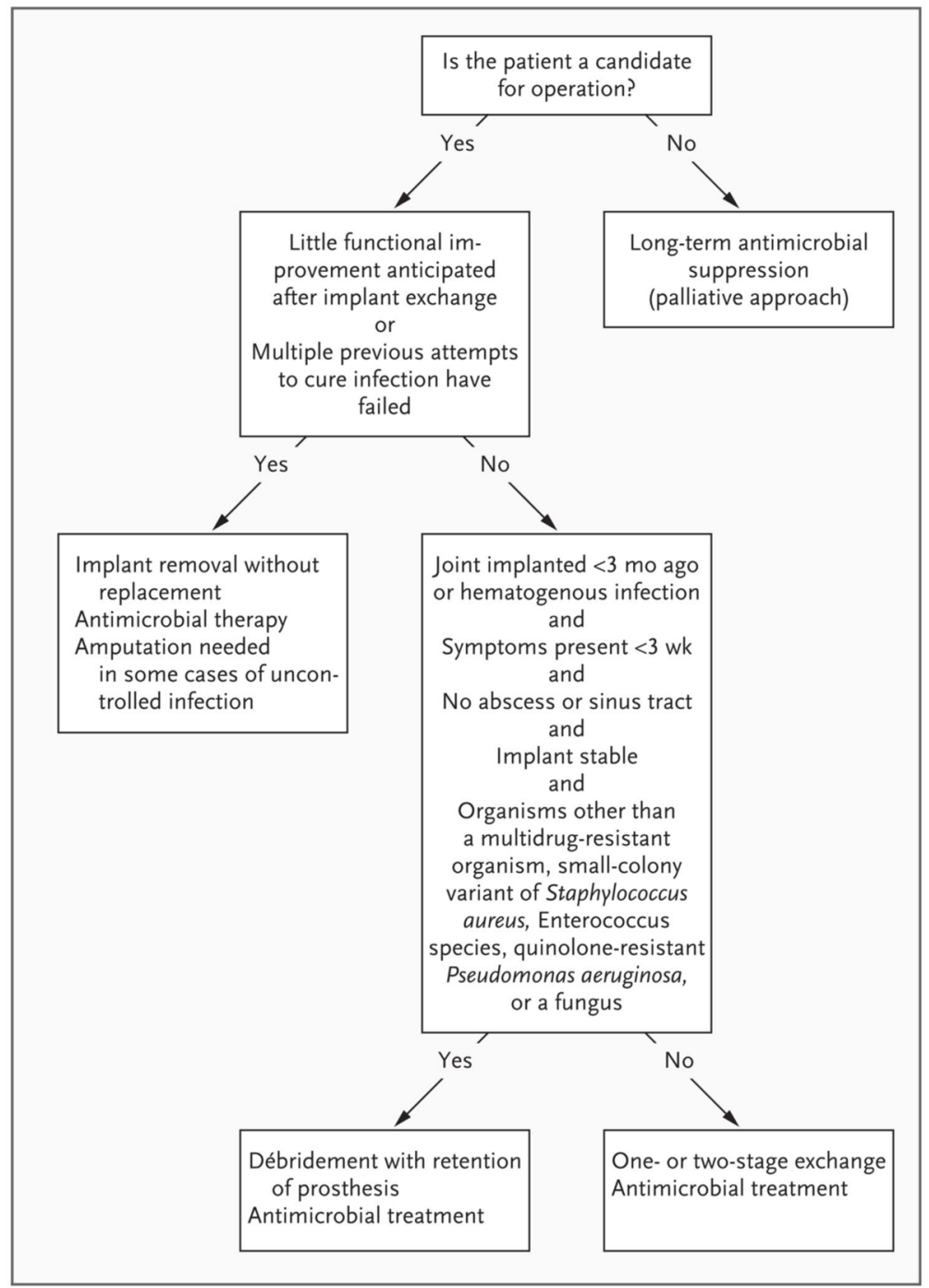

Figure 1.

Algorithm for the management of an infected prosthetic joint. [With permission from the Massachusetts Medical Society: Del Pozo et al. Infection Associated with Prosthetic Joints. New Engl J Med. 2009 Aug 20;361(8):787-94]. 